\title{
$C^{\infty}$ Ill-Posedness of the Mixed Problem for Hyperbolic Equations of Second Order
}

\author{
By
}

\section{Masaru TANIGUCH*}

\section{$\S 1$. Introduction}

We study the $C^{\infty}$ ill-posedness of the mixed problem for hyperbolic equations of second order

$$
\left\{\begin{array}{c}
L[u]=D_{t}^{2} u-2 \sum_{j=1}^{n} h_{\jmath}(t, x) D_{t} D_{x_{\jmath}} u-\sum_{i, j=1}^{n} a_{\imath \jmath}(t, x) D_{x_{\imath}} D_{x_{\jmath}} u+a_{0}(t, x) D_{t} u \\
+\sum_{j=1}^{n} a_{j}(t, x) D_{x_{\jmath}} u+d(t, x) u=f(t, x) \text { in } \Omega \\
\left.B[u]\right|_{x_{1}=0}=a_{11}\left(t, 0, x^{\prime}\right)^{-1 / 2} \\
\cdot\left\{a_{11}\left(t, 0, x^{\prime}\right) D_{x_{1}} u+\sum_{j=2}^{n} a_{1 j}\left(t, 0, x^{\prime}\right) D_{x_{\jmath}} u+h_{1}\left(t, 0, x^{\prime}\right) D_{t} u\right\} \\
+\sum_{j=2}^{n} b_{\jmath}\left(t, x^{\prime}\right) D_{x_{\jmath}} u-c\left(t, x^{\prime}\right)\left(1+\frac{h_{1}\left(t, 0, x^{\prime}\right)^{2}}{a_{11}\left(t, 0, x^{\prime}\right)}\right)^{1 / 2} \\
\cdot\left\{D_{t} u-\left(1+\frac{h_{1}\left(t, 0, x^{\prime}\right)^{2}}{a_{11}\left(t, 0, x^{\prime}\right)}\right)^{-1}\right. \\
\left.\cdot \sum_{j=2}^{n}\left(h_{\jmath}\left(t, 0, x^{\prime}\right)-\frac{h_{1}\left(t, 0, x^{\prime}\right)}{a_{11}\left(t, 0, x^{\prime}\right)} a_{i_{j}}\left(t, 0, x^{\prime}\right)\right) D_{x_{\jmath}} u\right\} \\
+\left.\gamma\left(t, x^{\prime}\right) u\right|_{x_{1}=0}=g\left(t, x^{\prime}\right) \text { in } \hat{\Omega}
\end{array}\right.
$$

where $x=\left(x_{1}, x_{2}, \cdots, x_{n}\right), x^{\prime}=\left(x_{2}, \cdots, x_{n}\right)(n \geqq 2), D_{t}=\partial / \partial t, D_{x_{j}}=\partial / \partial x_{j}, \Omega$ be an open neighborhood of $\left(t, x_{1}, x^{\prime}\right)=(0,0,0, \cdots, 0)$ in $\boldsymbol{R}^{1} \times \overline{\boldsymbol{R}_{+}^{1}} \times \boldsymbol{R}^{n-1}, \hat{\Omega}=\left\{\left(t, x^{\prime}\right) \mid\right.$ $\left.\left(t, 0, x^{\prime}\right) \in \Omega\right\}, T_{0}$ is a fixed positive number, $\Omega_{T_{0}}=\left\{\left(t, x_{1}, x^{\prime}\right) \in \Omega \mid \pm\left(t-T_{0}\right) \geqq 0\right\}$, the coefficients of operators $L$ and $B$ are smooth in $\Omega$ and $\hat{\Omega}$ respectively.

We assume the following conditions:

(A. I ) The operator $L$ is regularly hyperbolic in $\Omega_{T_{0}}^{+}, a_{11}\left(t, 0, x^{\prime}\right)>0$ in $\hat{\Omega}_{T_{0}}^{+}$ and $\hat{\Omega}_{T_{0}}^{+}$is connected where $\hat{\Omega}_{T_{0}}^{+}=\left\{\left(t, x^{\prime}\right) \mid\left(t, 0, x^{\prime}\right) \in \Omega_{T_{0}}^{+}\right\}$.

Communicated by S. Matsuura, December 22, 1987.

* Department of Mathematics, School of Science and Engineering, Waseda University, Tokyo 160, Japan. 
(A. II ) $\quad c\left(t, x^{\prime}\right) \neq-1$ in $\hat{\Omega}_{T_{0}}^{+}$.

(A.III) The quadratic equation

$$
\left(c\left(T, x_{0}^{\prime}\right)+1\right) z^{2}+2 b\left(T, x_{0}^{\prime}, \eta_{0}^{\prime}\right) z+c\left(T, x_{0}^{\prime}\right)-1=0
$$

has two roots $z_{1}$ and $z_{2}$ such that $\left|z_{2}\right|>1$ and $I_{m} z_{2} \neq 0$, where $x_{0}^{\prime}=\left(x_{02}, \cdots, x_{0 n}\right),\left(T, x_{0}^{\prime}\right) \in \hat{\Omega}_{T_{0}}^{+}, \eta_{0}^{\prime}=\left(\eta_{02}, \cdots, \eta_{0 n}\right) \in R^{n-1}-\{0\}$, and

$$
\left\{\begin{aligned}
& b\left(T, x_{0}^{\prime}, \eta_{0}^{\prime}\right)= \sum_{j=2}^{n} b_{j}\left(T, x_{0}^{\prime}\right) \eta_{0 j} / \tilde{d}\left(T, x_{0}^{\prime}, \eta_{0}^{\prime}\right) \\
& \tilde{d}\left(T, x_{0}^{\prime}, \eta_{0}^{\prime}\right)=\left[\sum_{i, \jmath=2}^{n} a_{\imath \jmath}\left(T, 0, x_{0}^{\prime}\right) \eta_{02} \eta_{0 j}-\frac{1}{a_{11}\left(T, 0, x_{0}^{\prime}\right)}\right. \\
& \cdot\left(\sum_{j=2}^{n} a_{1 j}\left(T, 0, x_{0}^{\prime}\right) \eta_{0 j}\right)^{2}+\left(1+\frac{h_{1}\left(T, 0, x^{\prime}\right)^{2}}{a_{11}\left(T, 0, x^{\prime}\right)}\right)^{-1} \\
&\left.\cdot\left(\sum_{j=2}^{n} h_{j}\left(T, 0, x^{\prime}\right) \eta_{0 j}-\frac{h_{1}\left(T, 0, x^{\prime}\right)}{a_{11}\left(T, 0, x^{\prime}\right)} \sum_{j=2}^{n} a_{1 j}\left(T, 0, x^{\prime}\right) \eta_{0 j}\right)^{2}\right]^{1 / 2}
\end{aligned}\right.
$$

Definition. We say that the mixed problem (1.1) is $C^{\infty}$ well-posed in $\Omega_{T_{0}}^{+}$ if the following conditions hold:

(i) For every $f \in C^{\infty}(\Omega)$ and $g \in C^{\infty}(\hat{\Omega})$ with $\operatorname{supp}[f] \subset \Omega_{T_{0}}^{+}$and $\operatorname{supp}[g] \subset \hat{\Omega}_{T_{0}}^{+}$, there exists $u$ in $C^{\infty}(\Omega)$ which satisfies (1.1).

(ii) Provided that $u \in C^{\infty}(\Omega)$, supp $[u] \subset \Omega_{T_{0}}^{+}, L[u]=0$ in $\Omega_{t_{0}}^{-}$and $\left.B[u]\right|_{x_{1}=0}=0$ in $\hat{\Omega}_{t_{0}}$ for every $t_{0}>T_{0}, \quad u=0$ in $\Omega_{t_{0}}$ where $\Omega_{t_{0}}=\left\{(t, x) \in \Omega \mid t \leqq t_{0}\right\}$ and $\hat{\Omega}_{t_{0}}=$ $\left\{\left(t, x^{\prime}\right) \mid\left(t, 0, x^{\prime}\right) \in \Omega_{t_{0}}^{-}\right\}$.

Now, we state our result:

Theorem. Assume the conditions (A. I ), (A. II) and (A.II). Then, the mixed problem (1.1) is not $C^{\infty}$ well-posed in $\Omega_{T_{0}}^{+}$.

By Wakabayashi's result [13: Theorem 1.4], we know that the Lopatinski determinant of the system $\left\{L^{0}, B^{0}\right\}$ is not zero if the problem (1.1) is $C^{\infty}$ wellposed, where $L^{0}$ and $B^{0}$ are the principal part of $L$ and $B$ in (1.1) respectively. In $[2],[8],[9],[10],[11]$, and [12], we discovered and developed the method to reduce the mixed problem for hyperbolic equations of second order to the one for symmetric hyperbolic pseudo differential systems of first order with nonnegative boundary condition or non-negative type boundary condition. In [3], Kreiss studied the Lopatinski determinant for the mixed problem for a simple hyperbolic system of first order with two space variables. Here, we can easily prove that the Lopatinski determinant $R\left(t, x^{\prime}, \tau, \eta^{\prime}\right)$ for the system $\left\{L^{0}, B^{0}\right\}$ is zero at $\left(T, x_{0}^{\prime}, \tau_{0}, \eta_{0}^{\prime}\right)$ or $\left(T, x_{0}^{\prime}, \tau_{0},-\eta_{0}^{\prime}\right)\left(\operatorname{Re} \tau_{0}>0\right)$ by the above systematization of the problem (1.1) and the similar method as the one in [3]. Therefore, we have the above theorem. 
Remark 1. Instead of $\Omega$, we can obtain the same result as our theorem for a domain $\Omega_{*}$ such that $\Omega_{*}$ is an open neighborhood of $(t, x)=(0,0)$ in $\boldsymbol{R}^{1} \times \overline{\boldsymbol{\omega}}$ and $\omega$ is a domain in $\boldsymbol{R}^{n}$ with smooth boundary.

Remark 2. We consider the mixed problem

$$
\begin{cases}L[u]=f & \text { in } \Omega \\ \left.B[u]\right|_{x_{1}=0}=g & \text { in } \hat{\Omega}\end{cases}
$$

where $(t, x)=\left(t, x_{1}, x^{\prime}\right) \in \Omega=\boldsymbol{R}^{1} \times \overline{\boldsymbol{R}_{+}^{1}} \times \boldsymbol{R}^{n-1},\left(t, x^{\prime}\right) \in \hat{\Omega}=\boldsymbol{R}^{1} \times \boldsymbol{R}^{n-1}, L$ and $B$ are the same operators in (1.1), the coefficients $h_{j}, a_{i j}, a_{0}, a_{j}$ and $d$ belong to $\mathscr{B}(\Omega)$ and are constant outside a compact set in $\Omega$, and the coefficients $b_{j}, c$ and $\gamma$ belong to $\mathscr{B}(\hat{\Omega})$ and are constant outside a compact set in $\hat{\Omega}$.

We assume the following condition for the problem (1.4):

(A. $\left.I^{\prime}\right) \quad$ The operator $L$ is regularly hyperbolic in $\Omega$ and $a_{11}\left(t, 0, x^{\prime}\right)>0$ in $\hat{\Omega}$.

Then, the mixed problem (1.4) is $L^{2}$ well-posed in $\Omega$ if and only if for every $\left(t, x^{\prime}\right) \in \hat{\Omega}$, the quadratic equation

$$
\left(c\left(t, x^{\prime}\right)+1\right) z^{2}+2 b\left(t, x^{\prime}, \eta^{\prime}\right) z+c\left(t, x^{\prime}\right)-1=0
$$

has roots in $\bar{D}=\{z \in \boldsymbol{C}|| z \mid \leqq 1\}$ if they are different and in $D=\{z \in C|| z \mid<1\}$ if they are equal.

We have the above fact by the Hermite theorem in [4] and the conformal mapping from the upper half plane to the unit disk (see [2]).

In [7: Remark 1.6], Taira treated the mixed problem for wave equation when the (1.5) has a double root in $D_{1}=\left\{z \in C \mid\left\{z \mid=1, I_{m} z \neq 0\right\}\right.$, and obtained a well-posedness of the problem.

Remark 3. In [6: Theorem 2], Soga considered the mixed problem for wave equation in a half space $(n \geqq 3)$ where the problem is $C^{\infty}$ well-posed in our sense and has the finite propagation property, and obtained the condition which give the ill-posedness of the problem. By Wakabayashi's result [13: Theorem 1.6] and the method of our proof of the theorem, we can easily obtain the same result as the one in [6].

Remark 4. When the coefficients of $L$ and $B$ are all constant and $\Omega=\boldsymbol{R}^{1} \times \overline{\boldsymbol{R}_{+}^{1}} \times \boldsymbol{R}^{n-1}$, we know a necessary and sufficient condition for $C^{\infty}$ wellposedness by Sakamoto [5]. In [1], Agemi and Shirota studied the mixed problem for wave equation in a half space precisely when $n=2, b_{2}$ and $c$ are real constants, and $\gamma=0$.

An outline of this paper is as follows. In $\S 2$, we treat the systematization of the wave equation. In $\S 3$, we give the proof of the theorem. 


\section{$\S 2$. Some Examples for Reduction to Symmetric Hyperbolic System}

In this section, we prepare the systematization of the wave equation $u_{t t}-u_{x x}-u_{y y}=0$, which have some parameters and enable us to study the Cauchy problem and the mixed problem for hyperbolic equations of second order by choosing parameters as appropriate functions and operators (see [2], [8], [9], $[10],[11],[12])$.

We set

$$
\begin{aligned}
& U=\left(\begin{array}{l}
U_{1} \\
U_{2}
\end{array}\right)=\left(\begin{array}{c}
u_{t}-u_{x}+z_{1} u_{y} \\
z_{1}\left(u_{t}+u_{x}\right)+u_{y}
\end{array}\right) \\
& V=\left(\begin{array}{l}
V_{1} \\
V_{2} \\
V_{3}
\end{array}\right)=\left(\begin{array}{c}
u_{t}-u_{x}+z_{2}\left(p_{2}-i q_{2}\right) u_{y} \\
z_{2}\left(u_{t}+u_{x}\right)+\left(p_{2}+i q_{2}\right) u_{y} \\
\sqrt{1+\left|z_{2}\right|^{2}} r_{2} u_{y}
\end{array}\right)
\end{aligned}
$$

and

$$
W=\left(\begin{array}{l}
W_{1} \\
W_{2} \\
W_{3}
\end{array}\right)=\left(\begin{array}{c}
u_{t}-u_{x}+z_{3}\left(p_{3}-i q_{3}\right) u_{y} \\
z_{3}\left(u_{t}+u_{x}\right)+\left(p_{3}+i q_{3}\right) u_{y} \\
u_{y}
\end{array}\right)
$$

where $u_{t t}-u_{x x}-u_{y y}=0, z_{1}, z_{2}$ and $z_{3}$ are complex constants, $p_{2}, q_{2}, p_{3}$ and $q_{3}$ are real constants with $p_{2}^{2}+q_{2}^{2}<1$ and $p_{3}^{2}+q_{3}^{2} \leqq 1$, and $r_{2}=\left(1-p_{2}^{2}-q_{2}^{2}\right)^{1 / 2}$.

Proposition 2.1. $U, V$ and $W$ satisfy the following equations respectively:

$$
U_{t}=\left(\begin{array}{rr}
-1 & 0 \\
0 & 1
\end{array}\right) U_{x}+\left(\begin{array}{ll}
0 & 1 \\
1 & 0
\end{array}\right) U_{y}=A_{1} U_{x}+B_{1} U_{y}
$$

$$
\left\{\begin{array}{l}
V_{t}=A_{2} V_{x}+B_{2} V_{y} \\
A_{2}=\left(\begin{array}{ccc}
-1 & 0 & 0 \\
0 & 1 & 0 \\
0 & 0 & \frac{1-\left|z_{2}\right|^{2}}{1+\left|z_{2}\right|^{2}}
\end{array}\right) \\
B_{2}=\left(\begin{array}{ccc}
0 & p_{2}-i q_{2} & \frac{r_{2}}{\sqrt{1+\left|z_{2}\right|^{2}}} \\
p_{2}+i q_{2} & 0 & \frac{z_{2} r_{2}}{\sqrt{1+\left|z_{2}\right|^{2}}} \\
\frac{r_{2}}{\sqrt{1+\left|z_{2}\right|^{2}}} & \frac{\bar{z}_{2} r_{2}}{\sqrt{1+\left|z_{2}\right|^{2}}} & \frac{-2\left(p_{2} \operatorname{Re} z_{2}+q_{2} \operatorname{Im} z_{2}\right)}{1+\left|z_{2}\right|^{2}}
\end{array}\right)
\end{array}\right.
$$

and

$$
\left\{\begin{array}{l}
M_{3} W_{t}=A_{3} W_{x}+B_{3} W_{y} \\
M_{3}=\left(\begin{array}{llc}
1 & 0 & 0 \\
0 & 1 & 0 \\
0 & 0 & \left(1+\left|z_{3}\right|^{2}\right) r_{3}
\end{array}\right), A_{3}=\left(\begin{array}{rcc}
-1 & 0 & 0 \\
0 & 1 & 0 \\
0 & 0 & \left(1-\left|z_{3}\right|^{2}\right) r_{3}
\end{array}\right)
\end{array}\right.
$$




$$
B_{3}=\left(\begin{array}{ccc}
0 & p_{3}-i q_{3} & r_{3} \\
p_{3}+i q_{3} & 0 & z_{3} r_{3} \\
r_{3} & \bar{z}_{3} r_{3} & -2 r_{3}\left(p_{3} \operatorname{Re} z_{3}+q_{3} \operatorname{Im} z_{3}\right)
\end{array}\right)
$$

where $r_{3}=1-p_{3}^{2}-q_{3}^{2}$.

Proof. By simple calculations, we obtain Proposition 2.1.

Q.E.D.

Remark 5. Let $M$ be

$$
\begin{aligned}
M= & D_{t}^{2}-2\left(\alpha_{1} D_{x_{1}}+\sum_{\jmath=2}^{n} \alpha_{\jmath} D_{x_{\jmath}}\right) D_{t} \\
& -\left(\beta_{11} D_{x_{1}}^{2}+2 \sum_{\jmath=2}^{n} \beta_{1 \jmath} D_{x_{1}} D_{x_{\jmath}}+\sum_{i, \jmath=2}^{n} \beta_{\imath_{\jmath}} D_{x_{\imath}} D_{x_{\jmath}}\right) .
\end{aligned}
$$

If $M$ is regularly hyperbolic with respect to $t$ and $\beta_{11}>0$, we obtain

$$
\left\{\begin{array}{l}
\sigma(M)=\tilde{\xi}^{2}+\widetilde{\widetilde{d}}\left(\eta^{\prime}\right)^{2}-\widetilde{\widetilde{\tau}}^{2} \\
\widetilde{\tilde{d}}\left(\eta^{\prime}\right)>0 \quad\left(\eta^{\prime} \neq 0\right)
\end{array}\right.
$$

which correspond to the symbol of the wave equation $\xi^{2}+\left|\eta^{\prime}\right|^{2}-\tau^{2} \quad\left(\eta^{\prime}=\left(\eta_{2}\right.\right.$, $\left.\left.\cdots, \eta_{n}\right)\right)$ where

$$
\left\{\begin{array}{l}
\tilde{\xi}=\frac{1}{\sqrt{\beta_{11}}}\left(\beta_{11} \xi+\sum_{j=2}^{n} \beta_{1 \jmath} \eta_{\jmath}+\alpha_{1} \tau\right) \\
\tilde{\tau}=\left(1+\frac{\alpha_{1}^{2}}{\beta_{11}}\right)^{1 / 2}\left\{\tau-\left(1+\frac{\alpha_{1}^{2}}{\beta_{11}}\right)^{-1}\left(\sum_{j=2}^{n} \alpha_{\jmath} \eta_{\jmath}-\frac{\alpha_{1}}{\beta_{11}} \sum_{\jmath=2}^{n} \beta_{1 \jmath} \eta_{\jmath}\right)\right\} \\
\tilde{\widetilde{d}}\left(\eta^{\prime}\right)=\left[\sum_{i, j=2}^{n} \beta_{\imath \jmath} \eta_{2} \eta_{\jmath}-\frac{1}{\beta_{11}}\left(\sum_{j=2}^{n} \beta_{1 \jmath} \eta_{\jmath}\right)^{2}\right. \\
\left.\quad+\left(1+\frac{\alpha_{1}^{2}}{\beta_{11}}\right)^{-1}\left(\sum_{\jmath=2}^{n} \alpha_{\jmath} \eta_{\jmath}-\frac{\alpha_{1}}{\beta_{11}} \sum_{j=2}^{n} \beta_{1 \jmath} \eta_{j}\right)^{2}\right]^{1 / 2}
\end{array}\right.
$$

for $\left(\xi, \eta^{\prime}\right) \in \boldsymbol{R}^{n}$.

\section{$\S 3$. Proof of the Theorem}

In this section, we shall prove the theorem.

Let $z_{1}$ and $z_{2}$ be two roots of the quadratic equation (1.2) such that $\left|z_{2}\right|>1$ and $\operatorname{Im} z_{2} \neq 0$. We set

$$
z_{2}=r e^{i \theta}(r>1,0<\theta<\pi \text { or } \pi<\theta<2 \pi)
$$

and 


$$
\left\{\begin{array}{l}
\tilde{\tau}_{ \pm}=\left(1+\frac{h_{1}\left(T, 0, x_{0}^{\prime}\right)^{2}}{a_{11}\left(T, 0, x_{0}^{\prime}\right)}\right)^{1 / 2}\left\{\tau \mp i\left(1+\frac{h_{1}\left(T, 0, x_{0}^{\prime}\right)^{2}}{a_{11}\left(T, 0, x_{0}^{\prime}\right)}\right)^{-1}\right. \\
\left.\quad \cdot\left(\sum_{j=2}^{n} h_{j}\left(T, 0, x_{0}^{\prime}\right) \eta_{0 j}-\frac{h_{1}\left(T, 0, x_{0}^{\prime}\right)}{a_{11}\left(T, 0, x_{0}^{\prime}\right)} \sum_{j=2}^{n} a_{1 j}\left(T, 0, x_{0}^{\prime}\right) \eta_{0 j}\right)\right\} \\
\tilde{d}\left(\eta_{0}^{\prime}\right)=\tilde{d}\left(T, x_{0}^{\prime}, \eta_{0}^{\prime}\right) \quad\left(=\tilde{d}\left(-\eta_{0}^{\prime}\right)\right) \\
\tau=\mu+i \sigma \quad\left(\mu>0, \sigma \in \boldsymbol{R}^{1}\right)
\end{array}\right.
$$

where $T, x_{0}, \eta_{0}^{\prime}$ and $\tilde{d}\left(T, x_{0}^{\prime}, \eta_{0}^{\prime}\right)$ are the same as in (1.3). Then, we have the Lopatinski determinant $R\left(T, x_{0}^{\prime}, \tau, \pm \eta_{0}^{\prime}\right)$ of the $\left\{L^{0}, B^{0}\right\}$ at $\left(T, x_{0}^{\prime}, \tau, \pm \eta_{0}^{\prime}\right)$,

$$
\begin{aligned}
R\left(T, x_{0}^{\prime}, \tau, \pm \eta_{0}^{\prime}\right) & =-\sqrt{\tilde{\tau}_{ \pm}^{2}+\tilde{d}\left(\eta_{0}^{\prime}\right)^{2}} \pm i \sum_{j=2}^{n} b_{j}\left(T, x_{0}^{\prime}\right) \eta_{0 j}-c\left(T, x_{0}^{\prime}\right) \tilde{\tau} \\
& =-\frac{c\left(T, x_{0}^{\prime}\right)+1}{2}\left(1+z_{2}\right)\left(\begin{array}{l}
\tilde{\tau}_{ \pm} \sqrt{\tilde{\tau}_{ \pm}^{2}+\tilde{d}\left(\eta_{0}^{\prime}\right)^{2}} \pm z_{1} i \tilde{d}\left(\eta_{0}^{\prime}\right) \\
z_{1}\left(\tilde{\tau}_{ \pm}-\sqrt{\tilde{\tau}_{ \pm}^{2}+\tilde{d}\left(\eta_{0}^{\prime}\right)^{2}}\right) \pm i \tilde{d}\left(\eta_{0}^{\prime}\right)
\end{array}\right)
\end{aligned}
$$

where $L^{0}$ and $B^{0}$ are the principal part of $L$ and $B$ in (1.1) respectively, and $\operatorname{Re}\left\{\sqrt{\tilde{\tau}_{ \pm}^{2}+\tilde{d}\left(\eta_{0}^{\prime}\right)^{2}}\right\}>0$. The systematization (2.1) is used in (3.3) (see [2]). From now on, we shall obtain a similar result as in [3: Lemma 2.2] and prove $R\left(T, x_{0}^{\prime}, \tau_{0}, \eta_{0}^{\prime}\right)=0$ or $R\left(T, x_{0}^{\prime}, \tau_{0},-\eta_{0}^{\prime}\right)=0$ for a $\tau=\tau_{0}\left(\operatorname{Re} \tau_{0}>0\right)$.

(i) The case $0<\theta<\pi$.

By $\tilde{d}\left(\eta_{0}^{\prime}\right)>0$, we can set

$$
\tilde{\tau}_{+}+\sqrt{\tilde{\tau}_{+}^{2}+\tilde{d}\left(\eta_{0}^{\prime}\right)^{2}}+z_{1} i \tilde{d}\left(\eta_{0}^{\prime}\right)=i(p+i q) \tilde{d}\left(\eta_{0}^{\prime}\right)
$$

where $p$ and $q$ are real numbers. Then, by (3.4), we get

$$
\begin{aligned}
& z_{1}\left(\tilde{\tau}_{+}-\sqrt{\tilde{\tau}_{+}^{2}+\tilde{d}\left(\eta_{0}^{\prime}\right)^{2}}\right)+i \tilde{d}\left(\eta_{0}^{\prime}\right) \\
& =\frac{1}{\tilde{d}\left(\eta_{0}^{\prime}\right)}\left[z_{1} \tilde{d}\left(\boldsymbol{\eta}_{0}^{\prime}\right)\left(\tilde{\tau}_{+}-\sqrt{\tilde{\tau}_{+}^{2}+\tilde{d}\left(\eta_{0}^{\prime}\right)^{2}}\right)\right]+i \tilde{d}\left(\eta_{0}^{\prime}\right) \\
& =\frac{1}{\tilde{d}\left(\eta_{0}^{\prime}\right)}\left[\left\{(p+i q) \tilde{d}\left(\boldsymbol{\eta}_{0}^{\prime}\right)+i\left(\tilde{\tau}_{+}+\sqrt{\tau_{+}^{2}+\tilde{d}\left(\boldsymbol{\eta}_{0}^{\prime}\right)^{2}}\right)\right\}\left(\tilde{\tau}_{+}-\sqrt{\tilde{\tau}_{+}^{2}+\tilde{d}\left(\boldsymbol{\eta}_{0}^{\prime}\right)^{2}}\right)\right]+i \tilde{d}\left(\eta_{0}^{\prime}\right) \\
& =(p+i q)\left(\tilde{\tau}_{+}-\sqrt{\tilde{\tau}_{+}^{2}+\tilde{d}\left(\boldsymbol{\eta}_{0}^{\prime}\right)^{2}}\right)+\frac{i}{\tilde{d}\left(\boldsymbol{\eta}_{0}^{\prime}\right)}\left[\tilde{\tau}_{+}^{2}-\tilde{\tau}_{+}^{2}-\tilde{d}\left(\boldsymbol{\eta}_{0}^{\prime}\right)^{2}\right]+i \tilde{d}\left(\boldsymbol{\eta}_{0}^{\prime}\right) \\
& =(p+i q)\left(\tilde{\tau}_{+}-\sqrt{\tilde{\tau}_{+}^{2}+\tilde{d}\left(\boldsymbol{\eta}_{0}^{\prime}\right)^{2}}\right) .
\end{aligned}
$$

By (3.3), (3.4) and (3.5), we have

$$
R\left(T, x_{0}^{\prime}, \tau, \eta_{0}^{\prime}\right)=-\frac{c\left(T, x_{0}^{\prime}\right)+1}{2}(p+i q)\left(1 z_{2}\right)\left(\begin{array}{c}
i \tilde{d}\left(\eta_{0}^{\prime}\right) \\
\tilde{\tau}_{+}-\sqrt{\tilde{\tau}_{+}^{2}+\tilde{d}\left(\eta_{0}^{\prime}\right)^{2}}
\end{array}\right) .
$$

By $i \tilde{d}\left(\eta_{0}^{\prime}\right)+z_{2}\left(\tilde{\tau}_{+}-\sqrt{\tilde{\tau}_{+}^{2}+\tilde{d}\left(\eta_{0}^{\prime}\right)^{2}}\right)=0$, we obtain 


$$
\begin{aligned}
\tilde{\tau}_{+} & =\frac{z_{2}^{2}+1}{2 i z_{2}} \tilde{d}\left(\boldsymbol{\eta}_{0}^{\prime}\right)=-\frac{i}{2}\left(z_{2}+\frac{1}{z_{2}}\right) \tilde{d}\left(\boldsymbol{\eta}_{0}^{\prime}\right) \\
& =-\frac{i}{2}\left[\left(r+\frac{1}{r}\right) \cos \theta+i\left(r-\frac{1}{r}\right) \sin \theta\right] \tilde{d}\left(\boldsymbol{\eta}_{0}^{\prime}\right) .
\end{aligned}
$$

Therefore, we have

$$
\operatorname{Re} \tilde{\tau}_{+}=\frac{1}{2}\left(r-\frac{1}{r}\right) \sin \theta \cdot \tilde{d}\left(\eta_{0}^{\prime}\right)>0 .
$$

Also, we get

$$
\left\{\begin{array}{l}
\sqrt{\tilde{\tau}_{+}^{2}+\tilde{d}\left(\eta_{0}^{\prime}\right)^{2}}=-\frac{i}{2}\left[\left(r-\frac{1}{r}\right) \cos \theta+i\left(r+\frac{1}{r}\right) \sin \theta\right] \tilde{d}\left(\eta_{0}^{\prime}\right) \\
\operatorname{Re}\left\{\sqrt{\tilde{\tau}_{+}^{2}+\tilde{d}\left(\eta_{0}^{\prime}\right)^{2}}\right\}>0 .
\end{array}\right.
$$

Then, we have $R\left(T, x_{0}^{\prime}, \tau_{0}, \eta_{0}^{\prime}\right)=0$ where $\tau_{0}$ is a complex number with a positive real part which is determined by (3.7).

(ii) The case $\pi<\theta<2 \pi$.

By $\tilde{d}\left(-\eta_{0}^{\prime}\right)=\tilde{d}\left(\eta_{0}^{\prime}\right)$, we have, at $\left(T, x_{0}^{\prime}, \tau,-\eta_{0}^{\prime}\right)$,

$$
\begin{aligned}
& R\left(T, x_{0}^{\prime}, \tau,-\eta_{0}^{\prime}\right)=-\sqrt{\tilde{\tau}_{-}^{2}+\tilde{d}\left(\eta_{0}^{\prime}\right)^{2}}-i \sum_{j=2}^{n} b_{j}\left(T, x_{0}^{\prime}\right) \eta_{0 j}-c\left(t, x_{0}^{\prime}\right) \tilde{\tau}_{-} \\
& =-\frac{c\left(T, x_{0}^{\prime}\right)+1}{2}\left(\begin{array}{ll}
1 & \left.z_{2}\right)
\end{array}\right)\left(\begin{array}{l}
\tilde{\tau}_{-}+\sqrt{\tilde{\tau}_{-}^{2}+\tilde{d}\left(\eta_{0}^{\prime}\right)^{2}}-z_{1} i \tilde{d}\left(\eta_{0}^{\prime}\right) \\
z_{1}\left(\tilde{\tau}_{-}-\sqrt{\tilde{\tau}_{-}^{2}+\tilde{d}\left(\eta_{0}^{\prime}\right)^{2}}\right)-i \tilde{d}\left(\eta_{0}^{\prime}\right)
\end{array}\right)
\end{aligned}
$$

where $\operatorname{Re}\left\{\sqrt{\tilde{\tau}_{-}^{2}+\tilde{d}\left(\eta_{0}^{\prime}\right)^{2}}\right\}>0$.

By $\tilde{d}\left(\eta_{0}^{\prime}\right)>0$, we can set

$$
\tilde{\tau}_{-}+\sqrt{\tilde{\tau}_{-}^{2}+\tilde{d}\left(\eta_{0}^{\prime}\right)^{2}}-z_{1} i \tilde{d}\left(\eta_{0}^{\prime}\right)=-i(p+i q) \tilde{d}\left(\eta_{0}^{\prime}\right) .
$$

Then, we have, by (3.11),

$$
\begin{aligned}
z_{1}\left(\tilde{\tau}_{-}-\sqrt{\tilde{\tau}_{-}^{2}+\tilde{d}\left(\eta_{0}^{\prime}\right)^{2}}\right)-i \tilde{d}\left(\boldsymbol{\eta}_{0}^{\prime}\right) & =\frac{1}{\tilde{d}\left(\eta_{0}^{\prime}\right)}\left[z_{1} \tilde{d}\left(\boldsymbol{\eta}_{0}^{\prime}\right)\left(\tilde{\tau}_{-}-\sqrt{\tilde{\tau}_{-}^{2}+\tilde{d}\left(\eta_{0}^{\prime}\right)^{2}}\right)\right]-i \tilde{d}\left(\eta_{0}^{\prime}\right) \\
= & (p+i q)\left(\tilde{\tau}_{-}-\sqrt{\tilde{\tau}_{-}^{2}+\tilde{d}\left(\boldsymbol{\eta}_{0}^{\prime}\right)^{2}}\right)
\end{aligned}
$$

By (3.11) and (3.12), we obtain

$$
R\left(T, x_{0}^{\prime}, \tau,-\eta_{0}^{\prime}\right)=-\frac{c\left(T, x_{0}^{\prime}\right)+1}{2}(p+i q)\left[-i \tilde{d}\left(\eta_{0}^{\prime}\right)+z_{2}\left(\tilde{\tau}_{-}-\sqrt{\tilde{\tau}_{-}^{2}+\tilde{d}\left(\eta_{0}^{\prime}\right)^{2}}\right)\right] .
$$

By $-i \tilde{d}\left(\boldsymbol{\eta}_{0}^{\prime}\right)+z_{2}\left(\tilde{\tau}_{-}-\sqrt{\tilde{\tau}_{-}^{2}+\tilde{d}\left(\eta_{0}^{\prime}\right)^{2}}\right)=0$, we get

$$
\tilde{\tau}_{-}=\frac{z_{2}^{2}+1}{-2 i z_{2}} \tilde{d}\left(\eta_{0}^{\prime}\right)=\frac{i}{2}\left[\left(r+\frac{1}{r}\right) \cos \theta+i\left(r-\frac{1}{r}\right) \sin \theta\right] \tilde{d}\left(\eta_{0}^{\prime}\right) .
$$

Therefore, we have 


$$
\operatorname{Re} \tilde{\tau}_{-}=-\frac{1}{2}\left(r-\frac{1}{r}\right) \sin \theta \cdot \tilde{d}\left(\eta_{0}^{\prime}\right)>0 .
$$

Also, we obtain $\operatorname{Re}\left\{\sqrt{\tilde{\tau}_{-}^{2}+\tilde{d}\left(\eta_{0}^{\prime}\right)^{2}}\right\}>0$. Then, we have $R\left(T, x_{0}^{\prime}, \tau_{0},-\eta_{0}^{\prime}\right)=0$ where $\tau_{0}$ is a complex number with a positive real part which is determined by (3.14).

By the above results and Wakabayashi's result [13: Theorem 1.4], we get our theorem.

Q.E.D.

\section{References}

[1] Agemi, R. and Shirota, T., On necessary and sufficient conditions for $L^{2}$-wellposedness of mixed problems for hyperbolic equations, J. Fac. Hokkaido Univ., 21 (1970), 133-151.

[2] Kojima, K. and Taniguchi, M., Mixed problem for hyperbolic equations in a domain with a corner, Funkcial. Ekvac., 23 (1980), 171-195.

[3] Kreiss, H.O., Boundary conditions for hyperbolic differential equations, Lecture Notes in Mathematics 363, Springer, 1974, 64-74.

[4] Miyatake, S., Mixed problem for hyperbolic equations of second order with first order complex boundary operators, Japan. J. Math., 1 (1975), 111-158.

[5] Sakamoto, R., $\mathcal{E}$-well posedness for hyperbolic mixed problems with constant coefficients, J. Math. Kyoto Univ., 14 (1974), 93-118.

[6] Soga, H., Mixed problems for the wave equation in a quarter space with a first order boundary condition, Osaka J. Math., 16 (1979), 271-288.

[7] Taira, K., On some non-coercive boundary value problems for the Laplacian, $J$. Fac. Scien. Univ. Tokyo, Sec. IA, 23 (1976), 343-367.

[8] Taniguchi, M., Mixed problem for hyperbolic equations of second order in a domain with a corner, Tokyo J. Math., 5 (1982), 183-211.

[9] —-, Mixed problem for weakly hyperbolic equations of second order with degenerate Neumann boundary condition, Funkcial. Ekvac., 27 (1984), 331-366.

[10] — Correction to: Mixed problem for weakly hyperbolic equations of second order with degenerate first order boundary condition, Tokyo J. Math., 9 (1986), 259-263.

[11] —, Mixed problem for weakly hyperbolic equations of second order with degenerate oblique boundary condition, Funkcial. Ekvac., 30 (1987), 351-382.

[12] - A systematization of the $L^{2}$-well-posed mixed problem and its applications to the mixed problem for weakly hyperbolic equations of second order, Funkcial. Ekvac., 31 (1988), 249-296.

[13] Wakabayashi, S., A necessary condition for the mixed problem to be $C^{\infty}$ well-posed, Comni. in Partial Differential Equations, 5 (1980), 1031-1064. 